\title{
Interaction between the Wilms tumour factor-1 element in the promoter of Amh and a downstream enhancer is required for a strong expression of the gene in pre-pubertal sertoli cells
}

\author{
David W. Dresser
}

The Ashworth Laboratory, The University of Edinburgh, Edinburgh, UK

Email: david.dresser@btinternet.com

Received 12 March 2013; revised 15 April 2013; accepted 10 June 2013

Copyright (C) 2013 David W. Dresser. This is an open access article distributed under the Creative Commons Attribution License, which permits unrestricted use, distribution, and reproduction in any medium, provided the original work is properly cited.

\begin{abstract}
Amh (anti-Müllerian hormone) is a single copy gene which is expressed strongly in Sertoli cells in the foetal testis and participates in the onset of sexual differentiation. Its promoter driving the expression of a reporter gene (d2EGFP) has been used to analyse the role of certain defined putative elements and a downstream enhancer element in gene expression. These experiments were carried out in vitro using a line of pre-pubertal mouse Sertoli cells, transienly transfected with circular DNA constructs with variously mutated promoter elements. A downstream enhancer element, situated immediately 3' of the polyadenylation (PA) signal for Amh, has been inserted in an equivalent position in the d2EGFP construct. When the Amh promoter is unmodified, the downstream enhancer (DE) is positively associated with a large increase in EGFP expression. This is at least partly the consequence of an increased rate of expression by individual cells. Experiments using variously truncated Amh promoters indicate that an upstream region $(-214$ to -336$)$ may play a minor role in facilitating enhancement. However mutation of the Wilms tumour factor-1 element, situated between the tata box and the start of translation, results in an almost complete suppression of enhancement.
\end{abstract}

Keywords: Mouse Cell Lines; In Vitro; Amh Promoter; SMAT; Pre-Pubertal Sertoli; Downstream Enhancer; Wilms Tumour Factor Element

\section{INTRODUCTION}

Amh, a member of the TGFbeta (BMP) family of trans- forming growth factor genes, plays a key role in early sexual differentiation in male mammals [1] and possibly a more subtle and protracted role in females [2]. Understanding the way in which gene expression is controlled in pre-Sertoli cells in the foetal testis, may help in decyphering the differentiation cascade triggered by the initial expression of Sry [3]. In the experiments to be described here the Amh promoter has been used to drive expression of a reporter gene (d2EGFP) in vitro, thus allowing a simple and quick way to investigate the role of constituent elements of the Amh promoter [4]. This approach largely confirms results obtained in vivo with an Amh promoter driving the expression of AMH [5-9]. It therefore seems likely that the in vitro system affords a convenient and economical approach to understanding other aspects of the control of gene expression by the Amh promoter.

Previously it was suggested that $1-3 \mathrm{~kb}$ of DNA immediately downstream of the Amhpolyadenylation (PA) signal, might play a role as a modulator of expression [10]. Further analysis of this region now shows that the 3' part of this sequence is in the open reading frame (ORF) of yet another gene in the cluster around Amh. This gene is transcribed in an anti-sense direction and codes for JP45 (Jsrp1-junctional sarcoplasmic reticulum protein [11]. JP45 runs from 15260 to 11973 in mouse genomic sequence X83733 - the Amh enhancer sequence (DE) runs from 11106 to 11195 .

The results described in this paper now confirm that 89nt immediately downstream of the Amh PA signal is effective as an enhancer of Amh promotion. The enhancement role of this stretch of DNA and its dependence on the Wilms Tumour factor-1 element (Wt) upstream in the Amh promoter, has been established but leaves as a matter of conjecture the exact molecular me- 
chanism of enhancement.

\section{MATERIALS AND METHODS}

SMAT cells (pre-pubertal Sertoli), TM4 cells (post-pubertal Sertoli) [12] and 3T3 (fibroblast) cells, are all mouse cell lines which adhere to tissue culture plasticware. They were grown in DMEM-F12 medium with glutamax and with $10 \%$ foetal calf serum plus penicillin and streptomycin. Assays were based on quadruplicate cultures in Costar 24-well plates $\left(\sim 2 \times 10^{5}\right.$ cells/well). Transfections with LipofectAmine 2000 were carried out in antibiotic free medium. Transfections for SMAT and $3 \mathrm{~T} 3$ cells were made with $800 \mathrm{ng}$ DNA and $2 \mu \mathrm{l} \mathrm{Li}-$ pofectAmine and TM4 cells with $1200 \mathrm{ng}$ DNA and $3 \mu \mathrm{l}$ Lipofect-Amine per well.

Details of the flow cytometric analysis of d2EGFP reporter gene expression and of the maintenance of the cell lines, have been described previously [4,10]. An index of EGFP (green) fluorescence was measured using aflowcytometer monitoring red (Iexas red) and green (fluorescein) channnels. This enables autofluorescence to be defined accurately to allow a window for cells expressing EGFP specific fluorescence to be measured accurately. The index of expression is the product of the number of cells in the green window expressed as percent of total live cells $(\%)$ and the geometric mean brightness $(\mathrm{Gm})$ of these cells $(\mathrm{I}=\% \times \mathrm{Gm})$.

Free DE and control DNA was prepared by synthesizing complimentary $90 \mathrm{nt}$ oligo-nucleotides which were annealed by mixing them in equal molar proportions, heating them to $98^{\circ}$ and allowing the mixture to cool very slowly overnight in a Dewar flask.

\section{RESULTS}

Previous results [4] hinted that the increased response index seen when an Amh promoter with a mutated proxGata element was used to drive expression of the reporter gene (d2EGFP), was explicable in terms of an increase in Gm. This implies that the mean expression per cell is increased. Figure 1 illustrates this point but also indicates that the same conclusion cannot be used to explain fully a decreased index with a mutated Se1 element.

Figure 2 illustrates the effectiveness of a downstream enhancer (DE) on responses driven by three different truncations of the Amh promoter in pre-pubertal Sertoli cells. Truncation X (see Figure 3) has virtually no effect while truncations $\mathrm{Y}$ and $\mathrm{Z}$ lead to a highly significant reduction in the level of EGFP expression. The presence of DE immediately 3' of the Amh PA signal, leads to a very large increase in the response driven by the intact (control) Amh promoter. The efficacy of DE on the responses driven by all three of the truncations is very much less. This result hints that something in the

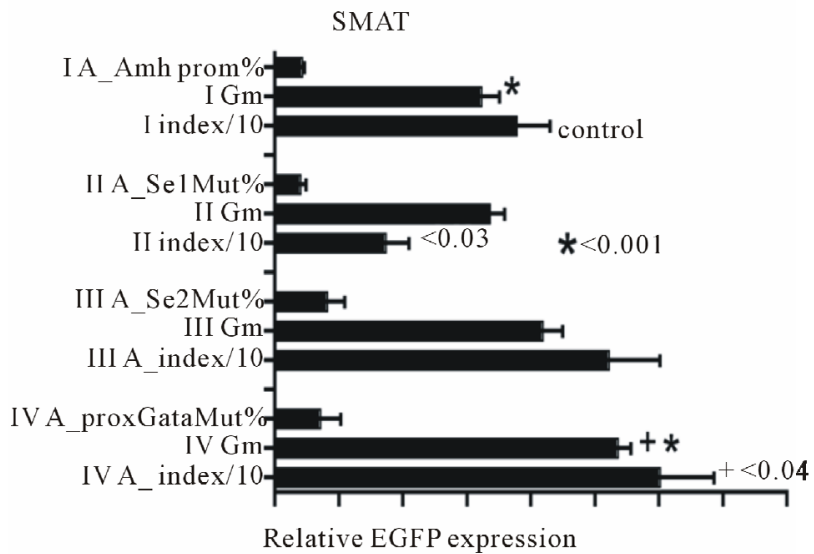

Figure 1. Data which confirms that an Amh promoter with a mutated Sel element shows a significantly lower response Index than the control group and an increased EGFP expression Index when the proxGata element was mutated. It is relevant to note that since the Index is the product of \% of cells in the green window and their geometric mean brightness $(\mathrm{Gm})$, it is interesting to note that d2EGFP gene expresses a version of EGFP with an intracellular half-life of 2 hours, consequently the Gm (brightness) value is a good approximation to a measurement of rate of synthesis. The increased index seen with the mutated proxGata element can be accounted for by a significant increase in $\mathrm{Gm}$ and hence an increase rate of synthesis per cell. The lower index value seen with the mutated Se1 element cannot be completely interpreted in the same manner.

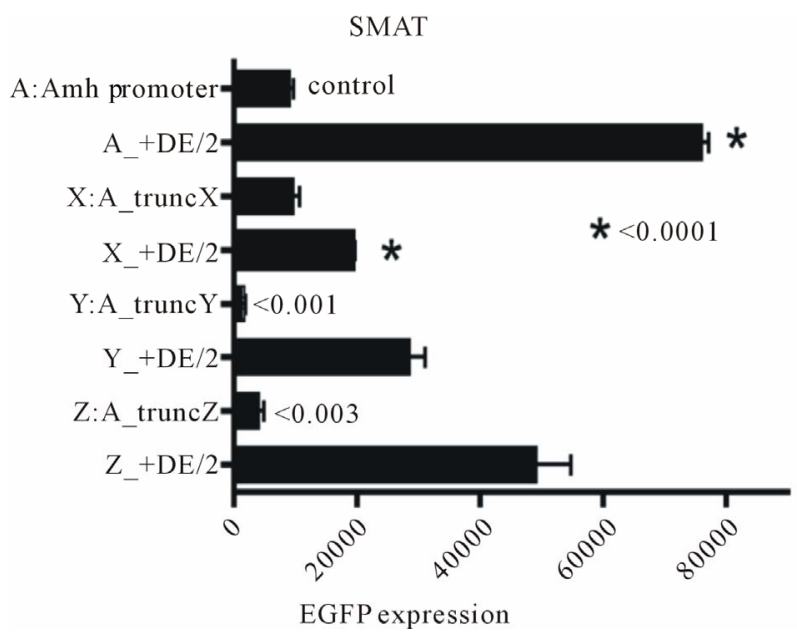

Figure 2. The results of an assay of the effect of three truncations of the Amh promoter and the differences seen when these modifications are combined with the presence of a downstream enhancer element (DE). Truncation X (for details see Figure 3) has virtually no effect on its own, whereas truncations $\mathrm{Y}$ and $\mathrm{Z}$ led to a profound reduction in expression. In sharp contrast when DE is present there is a significant reduction in the response seen in the TruncX + DE group implying that part of the promoter upstream of $\mathrm{tX}$ (see Figure 3 ) may play a role in the activity of DE. Never-the-less there is some increase in expression in all trunk + DE groups when these are compared with their respective truncations without DE. All $p$ values are from two tailed $t$ tests. The values of the columns marked/ 2 are halved to facilitate presentation. 


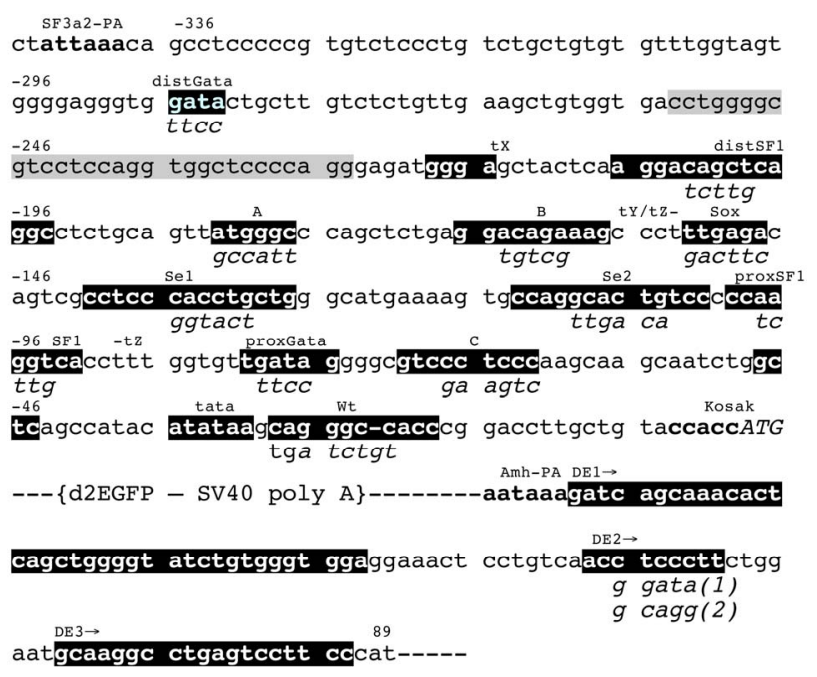

Figure 3. Nucleotide sequence (5' to 3') of a mouse Amh promoter. SF3a2-PA is the polyadenylation signal of an upstream gene coding for a spliceosome component [13]. Potential promoter elements are identified on the basis of sequence similarity with human, rat and other mammalian Amh promoter sequences: the order of elements is conserved. These potential elements are highlighted in black and identified by superscript titles, with mutated sequences indicated as subscripts. The grey highlighted sequence is an inverted repeat. Where possible the superscript titles are defined by their affinity for known transcription factors. Superscripts $\mathrm{tX}$ and $\mathrm{tY}$ indicate the start of two truncated Amh promoters terminating at the start of translation; $\mathrm{tZ}$ - and - $\mathrm{tZ}$ indicate the range of a third truncation. The start of translation (0) is position 8647 in GenBank genomic nucleotide sequence X83733. DE is a downstream enhancer starting at the polyadenylation signal for Amh. This 3' UTR element was inserted in the d2EGFP vector at a MluI site as indicated in this figure. The MluI site replaces an AflII site which was in the vector as supplied by Invitrogen.

Amh promoter upstream of the distSF1 element (Figure 3; -215 to -336 ) may play a small role in the action of DE.

Both Amh and the control minimal thymidine kinase $(\mathrm{Tk})$ promoter are ineffective when in reverse orientation. The presence of DE relatively increases responsiveness (Figure 4(a)), and it also is shown in part B that mutation of DE2 or replacement of the entire $89 \mathrm{nt}$ of the enhancer, completely reduces the effectiveness of the enhancer. This suggests that DE is nucleotide sequence specific and is not merely an inert spacer which could act by changing the flexibility of the DNA. Part $4 \mathrm{C}$ shows that the orientation of DE is not important and that DE can enhance expression driven by other promoters.

Figure 5 extends the results illustrated in Figure 4(a) and shows that the pattern of response in post-pubertal (TM4) Sertoli cells resembles that seen in 3T3 (fibroblast) cells and is different to that of pre-pubertal (SMAT) Sertoli cells. In the TM4 and 3T3 cells an Amh promoter in reverse orientation seems to do slightly better than the promoter in the forward orientation.

In Figure 6 a comparison is made between EGFP ex- (a)

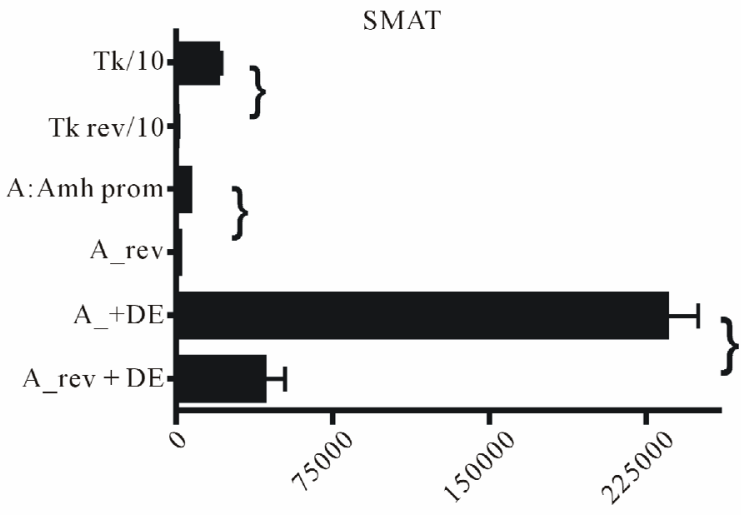

(b)

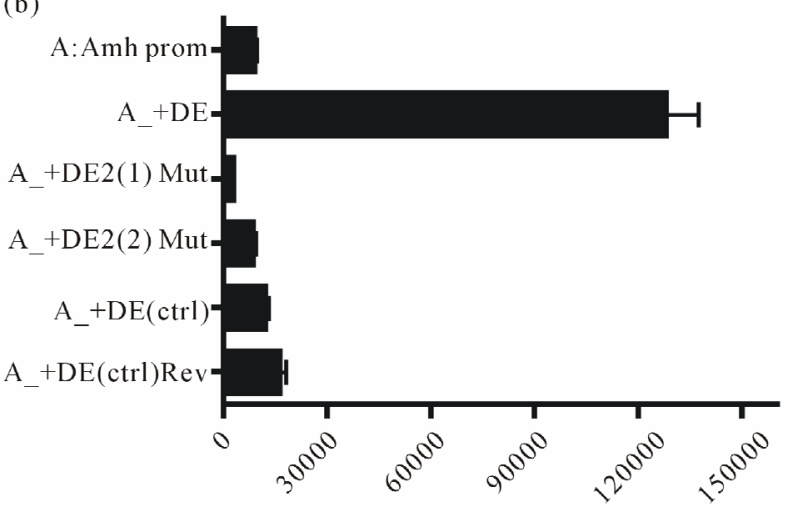

(c)

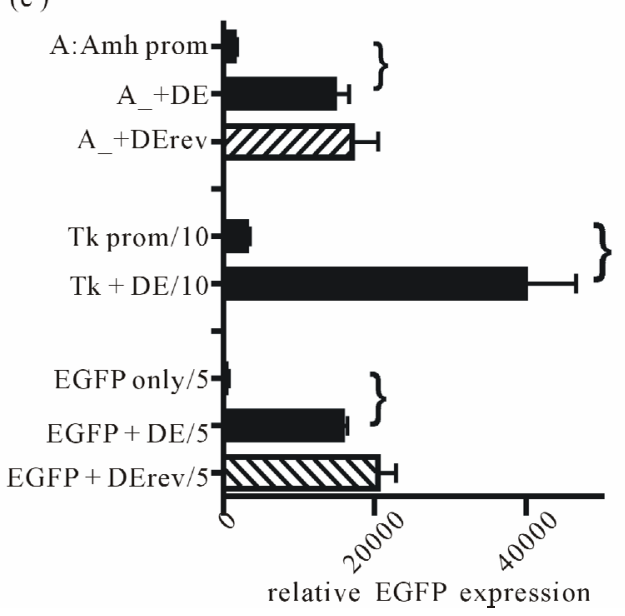

Figure 4. The effect of reversed orientation of promoter regions and the presence of the downstream enhancer (DE). (a) Reversing the orientation of promoters leads to a significant reduction in EGFP expression shown for each bracketed comparison $(p<0.0001)$. The presence of DE leads to significant increases in expression for the Amh promoter in either orientation; (b) Two different mutations of the DE2 site (see Figure 3) leads to a complete reduction in enhancer effectiveness. Attempts to mutate DE1 and DE3 were unsuccessful, although replacement of the entire 89 nucleotides with $90 \mathrm{nt}$ of a random sequence led to a complete reduction in expression; (c) Reversal of DE has no effect on its effectiveness as an enhancer. It is also clear that DE enhances expression driven by the Tk promoter and background expression "driven" by the MCS of EGFP. 


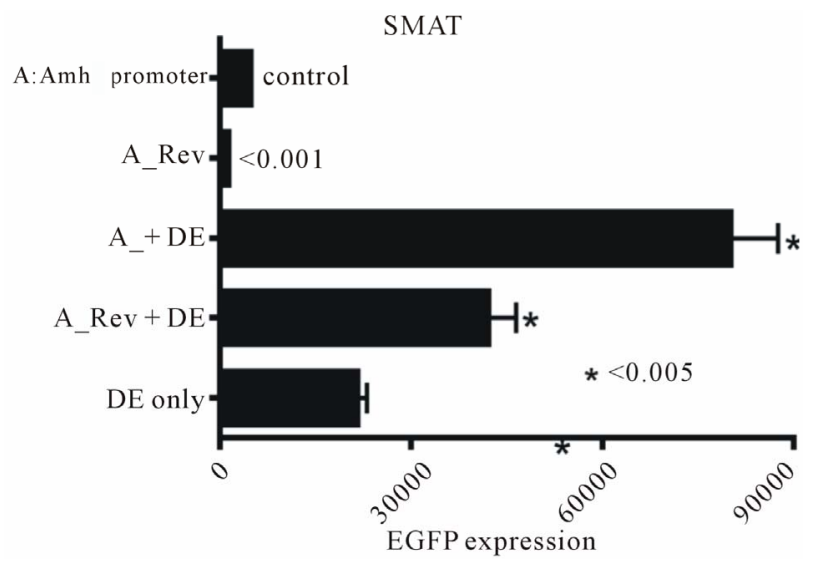

Figure 5. This figure illustrates the effectiveness of various combinations of Amh promoter and downstream enhancer (DE). EGFP expression was measured in three lines of mouse cells. The pattern of response in 3 T3 (fibroblast) and TM4 (post-pubertal Sertoli) cells are similar and are markedly different to that seen in SMAT (pre-pubertal Sertoli) cells. As in Figure 4 "DE only" is the response elicited by a pd2EGFP vector with a DE inserted 3' but lacking any 5' promoter. The MCS of this vector may have a weak non-specific promoterlike effect which can be reduced by truncation of the MCS.
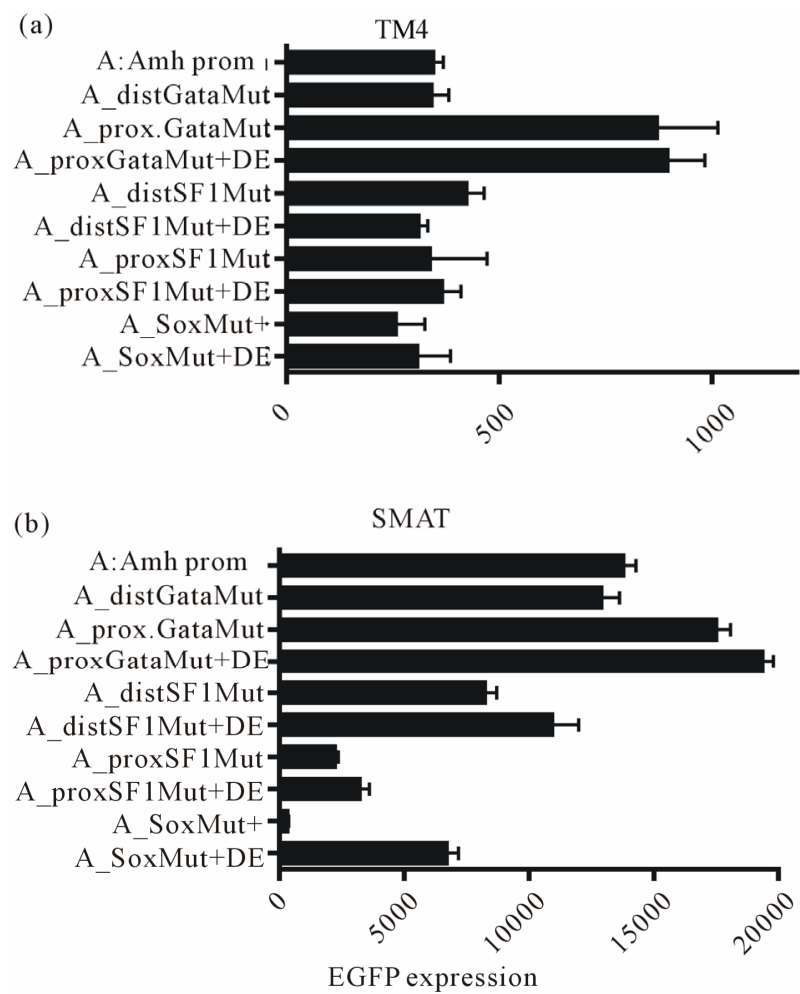

Figure 6. EGFP expression in TM4 (post-pubertal) and SMAT (pre-pubertal) Sertoli cells. Expression driven by a range of promoters with different elements mutated with and without an additional downstream enhancer element (DF). The presence of DE makes no difference in TM4 cells but in SMAT cells shows a small enhancement with the SF1 mutated elements. In contrast there is a significant enhancement of expression in the group with a mutated Sox element. pression in TM4 and SMAT cells clearly shows that DE makes little difference to responses elicited in TM4 cells by Amh promoters with variously mutated elements. However in SMAT cells there is confirmation of previous results showing that mutated distSF1, proxSF1 and Sox elements leads to reduced responses while a mutated proxGata element leads to an increase in expression.

In Figure 7(a) there is further confirmation of previous results obtained in SMAT cells [4], showing that mutated elements distSF1, Sox, Se1 and proxSF1 all lead to a reduced EGFP expression, while a mutated proxGata leads to an increased expression. It is also clear that mutated siteB, $\mathrm{Se} 2$ and $\mathrm{Wt}$ (Wilms tumour factor-1) elements have no effect when they are the sole mutated elements. However as shown in Figure 7(b), with the

(a)

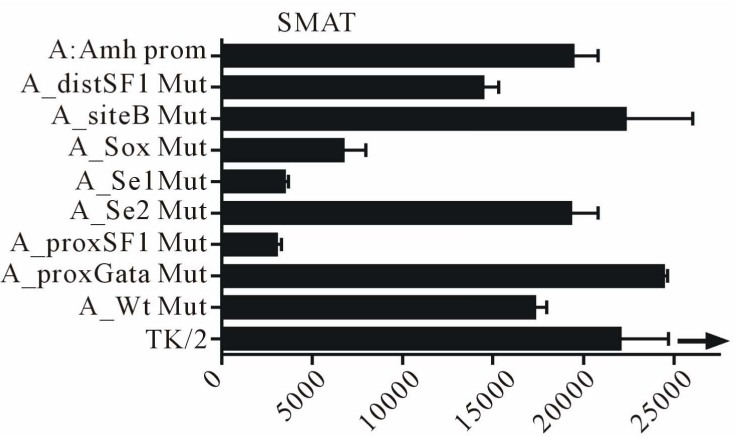

(b)

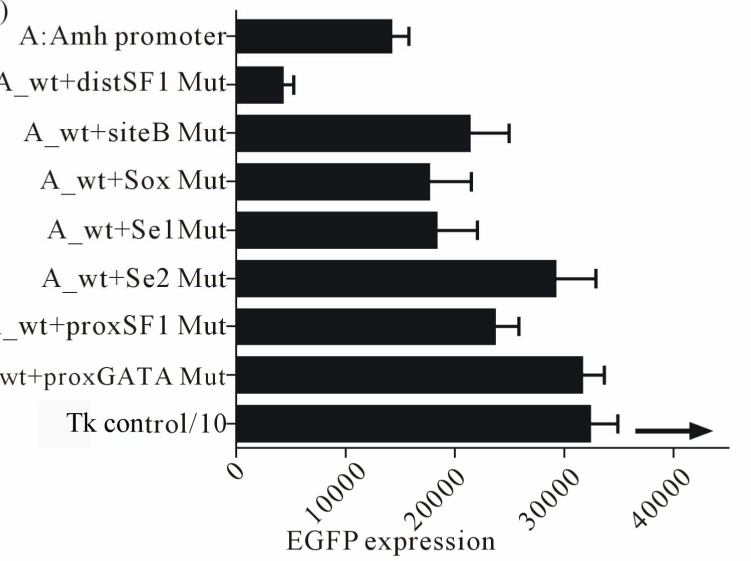

Figure 7. The effect of mutating individual elements of the Amh promoter driving the in vitro expression of EGFP in prepubertal Sertoli cells (SMAT). The UPPER part of the figure illustrates the effect of these mutations, confirming previously published data [4]. For example mutation of the Sox, Se1 and proxSF1 elements all resulted in a reduced expression, while a mutated proxGata resulted in an increased response index (see Figure 2). In the LOWER part each mutation is combined with a mutated Wilms tumour factor element (Wt). The addition of the Wt mutation seems to override the suppressive effect of Sox, Se1 and proxSF1 mutations, although the small suppression of promotion by distSF1 mutation alone is increased by the addition of a mutated Wt element. Since the experiments illustrated in the two parts of this figure were carried out with different batches of SMAT cells, on different occasions, therefore only relative comparisons can be made. 
exception of element distSF1, double mutation with the addition of a mutated $\mathrm{Wt}$ results in a reduction of both the negative and positive effects of single mutated elements recorded in part $\mathrm{A}$. The exception seems to be that distSF1 + Wt mutation leads to an augmention of suppression.

In Figure 8 combination of truncation $\mathrm{X}$ of the Amh promoter with mutated $\mathrm{Wt}$ and the presence of DE was tested in TM4 (post-pubertal) Sertoli cells and in SMAT (pre-pubertal) Sertoli cells. There is little measurable effect in TM4 cells but a significant reduction in the enhancer effect of DE in SMAT cells when Wt is mutated. This implies that an intact Wt element may be necessary for enhancement.

The experiment illustrated in Figure 9 extends the results shown in Figure 8 to show additionally that a mutated Wt is ineffective in permitting enhancement by DE in SMAT cells. Further confirmation of this observation is provided in Figures 10(a)-(f), where an intact Amhpromoter, and promoters with truncation $\mathrm{X}$ and truncation Y were used.

Addition of a relatively large amount of free double-stranded oligo-nucleotide DE DNA to the DNA-Li

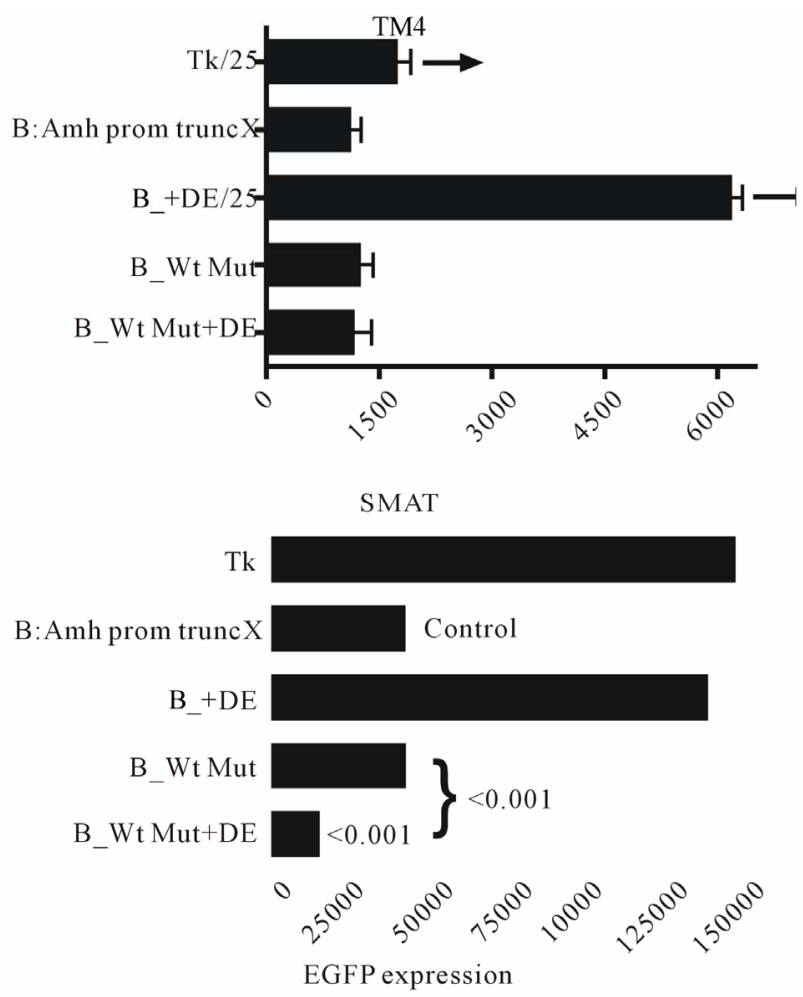

Figure 8. EGFP expression driven by a truncated Amh promoter in TM4 and SMAT Sertoli cells. The effect of adding the downstream enhancer (DE) in the presence of a mutaed or non-mutated Wilms tumour factor-1 (Wt) element. There is a strong suggestion from these results that in pre-pubertal Sertoli cells, DE requires the presence of an intact Wt element to be an effective enhancer.
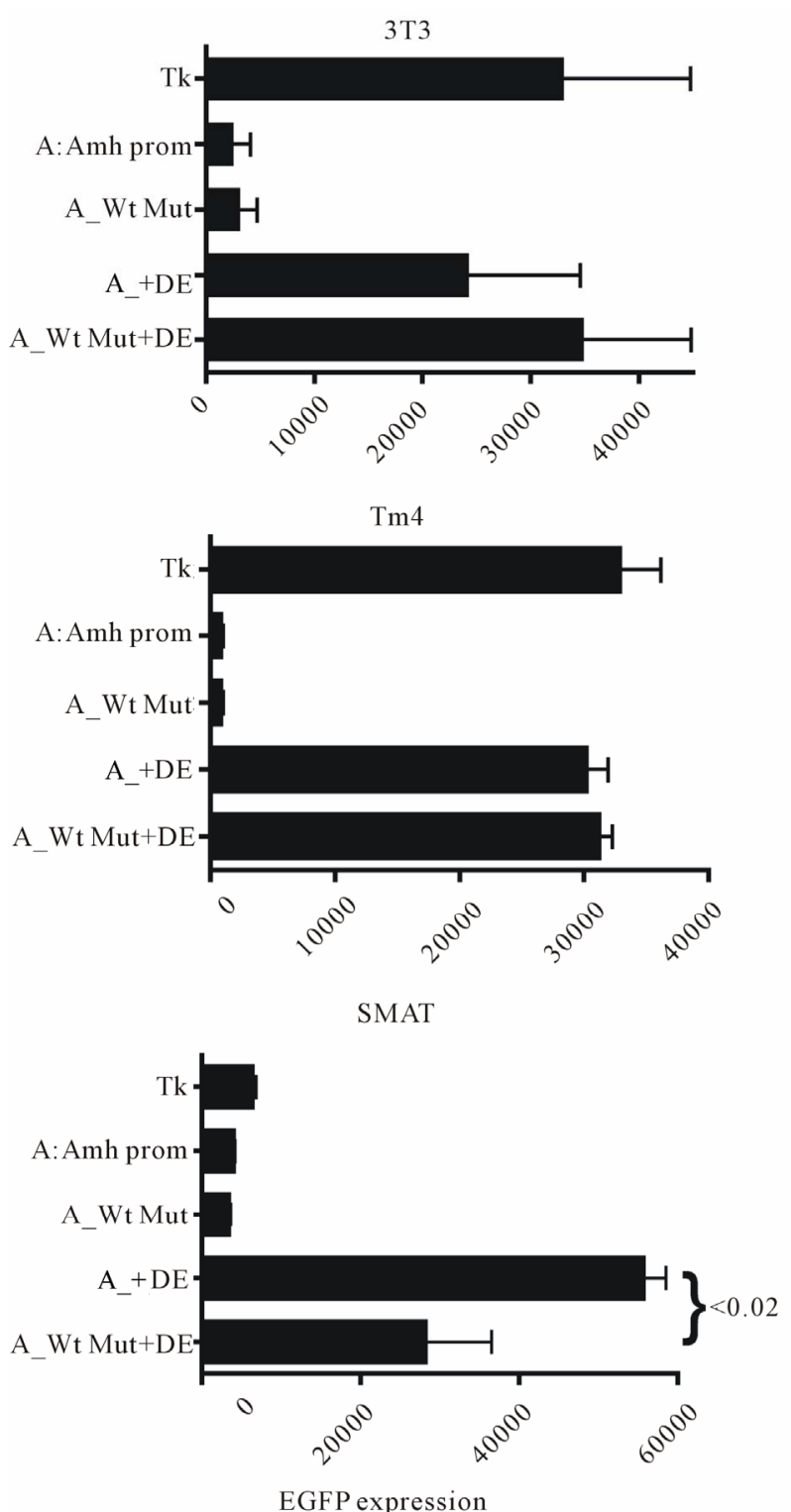

Figure 9. A comparison of in vitro EGFP expression responses in three lines of mouse cells. It can be seen that mutation of the Wilms tumour factor (Wt) element has on its own no effect in any of the cells tested, confirming previous observations. However in SMAT cells but not in either 3T3 or TM4 cells, it can be seen that Wt mutation shows a significant but not total, suppression of enhancement by the downstream element (DE). This result confirms the data in Figure 8 but using a "full length" Amh promoter.

pofectAmine mixture at the time of transfection, results in a significant specific lowering of expression. The results of these experiments are made available as a supplementary data file.

\section{DISCUSSION}

The results included in this paper, confirm those described earlier [4], and in addition establish that there is 

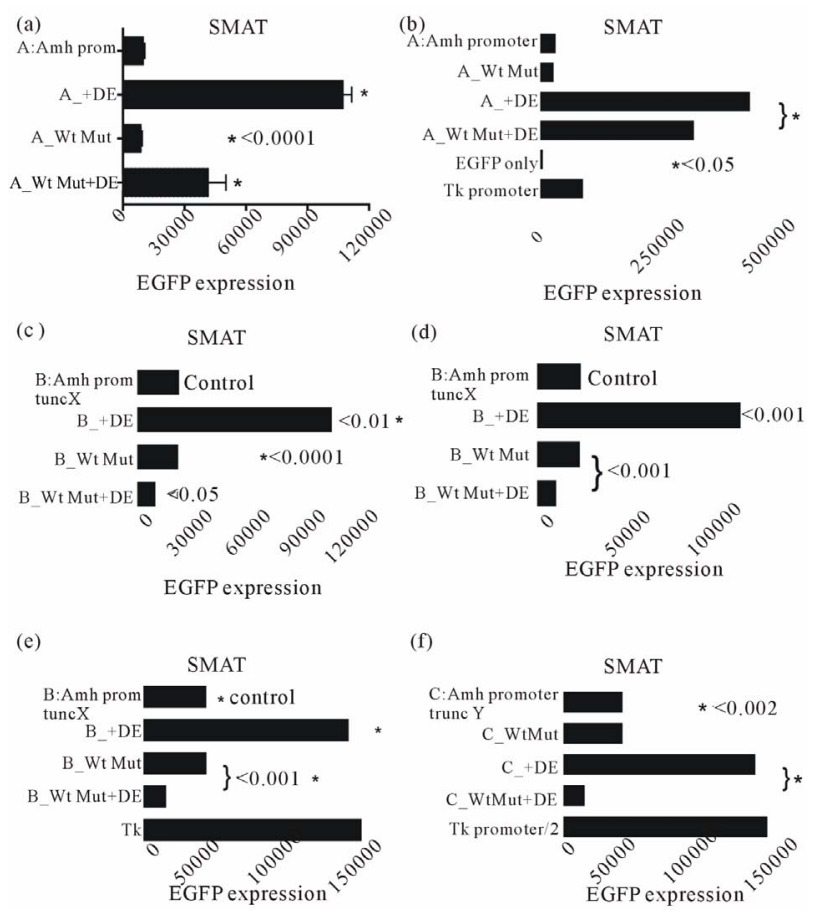

Figure 10. Six experiments were designed to illustrate the dependence of the downstream (3') enhancer on the upstream (5') Wilm's tumour factor. These experiments used a line of mouse pre-pubertal Sertoli cells (SMAT) with differently modified Amh promoters. This supplements the data in Figures 8 and $\mathbf{9}$. A mutated Wt significantly reduces enhancement by DE in all cases.

an enhancer (DE) situated immediately downstream of the PA signal for Amh. Furthermore it is shown that for full functionality this enhancer depends on the presence of an intact Wilms Tumour factor-1 element (Wt). Wt is situated in the promoter immediately upstream of the Amh gene and lies between the tata box and the start of translation. Interrogation of the PubMed data base shows that sequences with a close similarity to DE only occur immediately downstream of Amh/AMH genes. As shown in Figure 4 replacement of the DE sequence with a random DNA sequence of the same length, or mutation of the central portion of DE (DE2), led to a total ablation of enhancer activity. This suggests that enhancer function is both position and nucleotide sequence specific, although change of the orientation of DE makes no difference (Figure 4(c)).

As shown in Figure 2 analysis of enhancer (DE) activity in constructs with truncated Amh promoters indicates that a stretch of DNA at the 5' end of the promoter sequence, as illustrated in Figure 3 ( -336 to -215$)$, plays a small but significant role in enhancement. The assumption that position -336 is the effective 5' end of the mouse Amh promoter is based on the observation that immediately upstream is the polyadenylation (PA) signal for $\mathrm{SF} 3 \mathrm{a} 2$ which codes for a spliceosome component
[13]. It therefore seems unlikely but not impossible, that there are any Amh promoter elements upstream of this point.

In Figure 6 it can be seen that DE while functional in pre-pubertal Sertoli cells, is only weakly active in postpubertal Sertoli cells (TM4). Never-the-less DE has a measurable enhancement on the expression of EGFP driven by the Tk promoter in SMAT (Figure 4(c)). Other experiments, including those illustrated in Figure 9, suggest that DE is not functional in $3 \mathrm{~T} 3$ (mouse fibroblast) cells. This data and that mentioned in the previous paragraph, suggest that DE enhancement is dependent on a "pre-pubertal" cellular environment, possibly involving a cocktail of transcription factors specific to this point in development. Furthermore enhancement is also dependent on the presence of an intact Wilms tumour factor element $(\mathrm{Wt})$. A question therefore arises as to the molecular mechanism of enhancement. The results summariesed in Figure 4(b) support the view that enhancement depends on the DE2 sequence, which might be a specific anchor point which is instrumental in the folding back of the entire $\mathrm{DE}$ close to the promoter at the $\mathrm{Wt}$ element. Such a folding across $4-5 \mathrm{~kb}$ of ORF may be mediated by a duplex transcription-like bridging factor [14], with specificity for both DE2 and Wt. There is no hint in the DNA itself that sequence complementarity plays a role in such an interaction. The relatively small specific inhibition of DE activity by free DE DNA, introduced at the time of transfection (see Supplementary Data) (Figures S1 and S2), is compatible with this view but does not rule out other possible mechanisms [15-19]. For example once $\mathrm{DE}$ is juxtaposed close to $\mathrm{Wt}$, part of DE (DE1; DE3) might take on the role of supplementary promoter elements. It is not clear how this could affect the efficiency of use or recycling of mRNA. The results summarised in Figures 4(a) and (c) show that enhancement is sensitive to the orientation of the promoter but not to that of DE. This is compatible with the view that the promoter is an ordered assembly template and DE is an anchor point for a bridge.

Elements in the 3' UTR of several genes have been shown to influence the stability of transcripts by controling the number of adenines added to the tail of the mRNA - the longer the poly-A tail the more stable the message $[20,21]$. Also there is a report of a synergistic relationship between the poly-A tail and the cap (start of transcription) [22]. The 3' UTR is usually considered to be the DNA downstream of the terminator of translation but upstream of the PA signa: this DNA is transcribed into the message and presumably acts at that level. In contrast DE is situated downstream of the Amh PA signal and as mentioned above, when folded back onto $\mathrm{Wt}$, may act as a supplementary promoter element. In general the promoter can be seen as controlling the assem- 
bly of the constituents of the transcription mechanism. The Wt element lies between the tata box and the start of translation - this region may include different cap sites leading to alternative transcripts which may play a part in control of gene expression. There may be many other potential examples which have a role in the control of gene expression during sex development [23,24].

\section{ACKNOWLEDGEMENTS}

I thank Dr. N. di Clemente for providing the SMAT (prepubertalSertoli) cells; the University of Edinburgh for an Emeritus Fellowship; Professor Rick Maizels and his colleagues for the hospitality of the lab; and Daniel Guerrier for a critical reading of a draft manuscript.

\section{REFERENCES}

[1] Josso, N., Picard, J.Y., Rey, R. and di Clemente, N. (2006) Testicular anti-Mullerian hormone: History, genetics, regulation and clinical applications. Pediatric Endocrinology Reviews, 3, 347-358.

[2] Durlinger, A.L., Visser, J.A. and Themmen, A.P. (2002) Regulation of ovarian function: The role of anti-Mullerian hormone. Reproduction, 124, 601-609. doi:10.1530/rep.0.1240601

[3] Koopman, P., Gubbay, J., Vivian, N., Goodfellow, P. and Lovell-Badge, R. (1991) Male development of chromosomally female mice transgenic for Sry. Nature, 351, 117-121. doi:10.1038/351117a0

[4] Dresser, D.W. (2012) Mutated elements of a complex promoter (Amh) can help to demonstrate the role of certain elements in controlling differential gene expression. American Journal of Molecular Biology, 2, 351-358. doi:10.4236/ajmb.2012.24036

[5] de Santa Barbara, P., Moniot, B., Poulat, F. and Berta, P. (2000) Expression and subcellular localization of SF-1, SOX9, WT1, and AMH proteins during early human testicular development. Developmental Dynamics, 217, 293-298. doi:10.1002/(SICI)1097-0177(200003)217:3<293::AIDDVDY7>3.0.CO;2-P

[6] Oreal, E., Mazaud, S., Picard, J.Y., Magre, S. and CarreEusebe, D. (2002) Different patterns of anti-Mullerian hormone expression, as related to DMRT1, SF-1, WT1, GATA-4, Wnt-4, and Lhx9 expression, in the chick differentiating gonads. Developmental Dynamics, 225, 221232. doi: $10.1002 /$ dvdy. 10153

[7] Arango, N.A., Lovell-Badge, R. and Behringer, R.R. (1999) Targeted mutagenesis of the endogenous mouse Mis gene promoter: In vivo definition of genetic pathways of vertebrate sexual development. Cell, 99, pp. 409-419. doi:10.1016/S0092-8674(00)81527-5

[8] Münsterberg, A. and Lovell-Badge, R. (1991) Expression of the mouse anti-mullerian hormone gene suggests a role in both male and female sexual differentiation. Development, 113, 613-624.

[9] Schepers, G., Wilson, M., Wilhelm, D. and Koopman, P. (2003) SOX8 is expressed during testis differentiation in mice and synergizes with SF1 to activate the Amh promoter in vitro. Journal of Biological Chemistry, 278, 28101-28108. doi:10.1074/jbc.M304067200

[10] Dresser, D.W. and Guerrier, D. (2005) Candidate sertoli cell specific promoter element for a TGFbeta family member (Amh) and a 3' UTR enhancer/repressor for the same gene. Gene, 363, 159-165. doi:10.1016/j.gene.2005.08.004

[11] Anderson, A.A., Treves, S., Biral, D., Betto, R., Sandonà, D., Ronjat, M. and Zorzato, F. (2003) The novel skeletal muscle sarcoplasmic reticulum JP-45 protein. Molecular cloning, tissue distribution, developmental expression, and interaction with alpha 1.1 subunit of the voltagegated calcium channel. Journal of Biological Chemistry, 278, 39987-39989. doi:10.1074/jbc.M305016200

[12] Belville, C., Jamin, S.P., Picard, J.Y., Josso, N. and di Clemente, N. (2005) Role of type I receptors for antiMullerian hormone in the SMAT-1 Sertoli cell line. Oncogene, 24, 4984-4992.

[13] Dresser, D.W., Hacker, A., Lovell-Badge, R. and Guerrier, D. (1995) The genes for a spliceosome protein (Sap $62)$ and the anti-Mullerian Hormone are contiguous. Human Molecular Genetics, 4, 613-618. doi: $10.1093 / \mathrm{hmg} / 4.9 .1613$

[14] Anderson, A.M., Weasner, B.M., Weasner, B.P. and Kumar, B.P. (2012) Dual transcriptional activities of SIX define their roles in normal and ectopic eye development. Development, 139, 991-1000. doi:10.1242/dev.077255

[15] Miyamota, Y., Taniguchi, H., Hamel, F., Silversides, D.W. and Viger, R. (2008) A GATA4/WT1 cooperation regulates transcription of genes required for mammalian sex derermination and differentiation. BMC Molecular Biology, 9, 44-62. doi:10.1186/1471-2199-9-44

[16] Viger, R.S., Taniguchi, H., Robert, N.M. and Tremblay, J.J. (2004) Role of the GATA family of transcription factors in andrology. Journal of Andrology, 25, 441-452.

[17] Klattig, J., Sierig, R., Kruspe, D., Besenbeck, B. and Englert, C. (2007) Wilms' tumor protein Wt1 is an activator of the anti-Mullerian hormone receptor gene Amhr2. Molecular and Cellular Biology, 27, 4355-4364. doi:10.1128/MCB.01780-06

[18] Guittot, S.M., Tetu, A., Legault, E., Pilon, N., Silversides, D.W. and Viger, R.S. (2007) The proximal Gata4 promoter directs reporter gene expression to sertoli cells during mouse gonadal development. Biology of Reproduction, 76, 85-95. doi:10.1095/biolreprod.106.055137

[19] Sekido, R. and Lovell-Badge, R. (2008) Sex determination involves synergistic action of SRY and SF1 on a specific Sox 9 enhancer. Nature, 453, 930-934. doi:10.1038/nature06944

[20] Merritt, C., Rasojoson, D., Ko, D. and Seydoux, G. (2008) 3' UTR are the primary regulators of gene expression in the C. elegansgermline. Current Biology, 14, 1476-1482. doi:10.1016/j.cub.2008.08.013

[21] Derrigo, M., Cestelli, A., Savettieri, G. and diLiegro, I. (2000) RNA-protein interactrions in the control of stability and localization of messenger RNA. International Journal of Molecular Medicine, 5, 111-123.

[22] Gallie, D.R. (1991) The cap and poly(A) tail function 
synergistically to regulate mRNA translational efficiency. Genes \& Development, 5, 2108-2116. doi:10.1101/gad.5.11.2108

[23] White, S. and Sinclair, A. (2012) The molecular basis of gonadal development and disorders of sex development. Chapter 1 in J. M. Hutson, et al., Eds., Disorders of Sex Development, Springer, Berlin, pp. 1-9.

\section{SUPPLEMENTARY DATA}

Experiments with a mutated downstream enhancer (DE) and the Wilms tumour element (Wt) in the upstream Amh promoter, suggest that nucleotide sequence specificity is key in the interaction. Therefore experiments were carried out to check the effect of free DE DNA on the "enhancer effect".

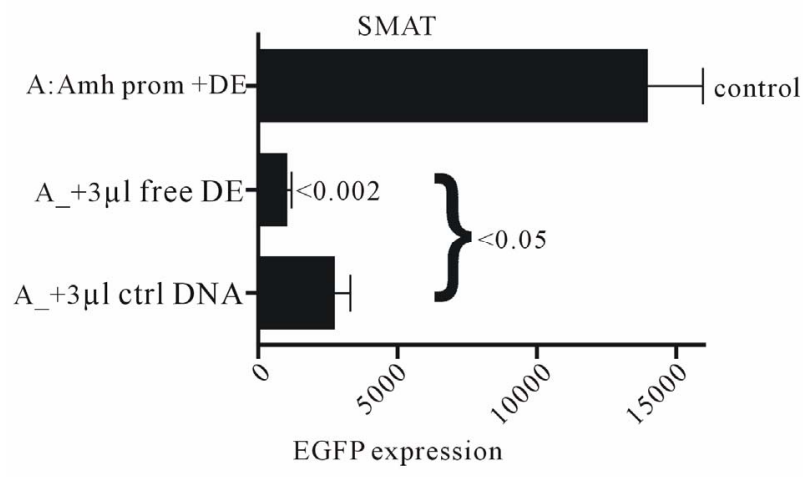

Figure S1. Free 89 nt DE DNA and a control DNA (ctrl) were added to cultures of SMAT cells, together with the modified Amh-EGFP-DE DNA expression vector $(\sim 4.3 \mathrm{~kb})$ and lipofectAmine 2000, at the time of transfection. There is an indication that $3 \mu \mathrm{l}$ of $50 \mathrm{pMol} / \mu \mathrm{l}$ of free DE DNA specifically interferes with expression of EGFP $(p<0.05)$.

\section{doi:10.1007/978-3-642-22964-0_1}

[24] Furuhata, A., Murakami, M., Ito, H., Yoshida, K., Sobue, S., Kikuchi, R., Iwasaki, T., Takagi, A., Kojima, T., Suzuki, M., Abe, A. and Murate, T. (2009) GATA-1 and GATA-2 binding to 3' enhancer of WT1 gene is essential for its transcription in acuteleukemia and solid tumor cells. Leukemia, 23, 1270-1277. doi:10.1038/leu.2009.13

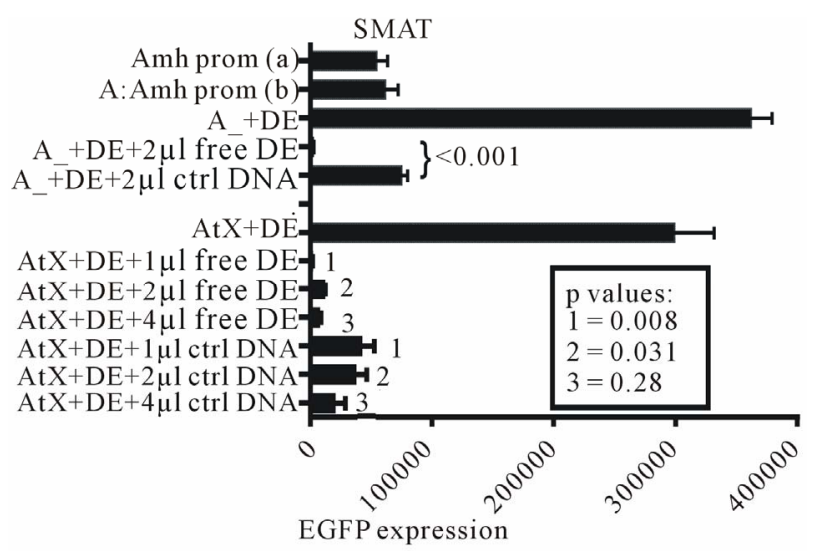

Figure S2. Experiments which confirm that free DE DNA interferes with the interaction of DE with the Wilms tumour factor element in the promoter of Amh used to drive the expression of the reporter gene (EGFP). Two independently prepared control Amh promoter constructs, (a) and (b), are included. The addition of $2 \mu \mathrm{l}$ of $50 \mathrm{pMol} / \mu 1 \mathrm{DNA}$ to the transfection mixture resulted in a significant reduction in EGFP expression. A similar experiment using a truncated Amh promoter (truncation X; AtX) showed broadly similar results. 